\title{
Current status and future directions in lipid management: emphasizing low-density lipoproteins, high-density lipoproteins, and triglycerides
} as targets for therapy

\author{
This article was published in the following Dove Press journal: \\ Vascular Health and Risk Management \\ II February 2010 \\ Number of times this article has been viewed
}

Yun Lin

\section{Shaymaa S Mousa \\ Nabil Elshourbagy \\ Shaker A Mousa}

The Pharmaceutical Research Institute, Albany College of Pharmacy and Health Sciences, Albany, NY, USA
Correspondence: Shaker A Mousa Professor of Pharmacology, Executive VP, and Chairman Pharmaceutical Research Institute, Albany College of Pharmacy and Health Sciences, One Discovery Drive,

Rensselaer, NY 12144, USA

Tel +l 5l 8-694-7397

$\mathrm{Fax}+\mathrm{I}$ 5I8-694-7567

Email shaker.mousa@acphs.edu

\begin{abstract}
Current lipid management guidelines are focused on decreasing low-density lipoprotein (LDL-C) levels as the primary target for reducing coronary heart disease (CHD) risk. Yet, many recent studies suggest that low levels of high-density lipoprotein (HDL-C) are a major independent risk factor for cardiovascular diseases. According to several clinical trials, a $1 \%$ increase in HDL-C is associated with a $0.7 \%-3 \%$ decrease in CHD events. The direct link between high levels of triglycerides (TG) and CHD, on the other hand, is less well defined. A large reduction in TG is needed to show a difference in CHD events, especially in men. Evidence for a shift in lipid management toward targeting both LDL-C and HDL-C as primary targets for therapy is presented. Currently, the 3-hydroxy-3-methylgutaryl coenzyme A reductase inhibitors (HMG-CoA reductase inhibitors) have proven to significantly decrease LDL-C levels, reduce CHD morbidity/mortality and improve overall survival. However, improvement of survival with statins may be due to other pleiotropic effects beyond LDL-C lowering. Fibric acid derivatives and niacin are primarily used to increase HDL-C levels, although with side effects. Future therapies targeting HDL-C may have profound results on reducing CHD morbidity and mortality. This article highlights existing and future targets in lipid management and is based on available clinical data. There is an urgent need for new treatments using a combination of drugs targeting both LDL-C and HDL-C. Such treatments are expected to have a superior outcome for dyslipidemia therapy, along with TG management.
\end{abstract}

Keywords: cholesterol, lipid, LDL-C, HDL-C, triglycerides, statin, nicotinic acid, fibric acid, atherosclerosis, coronary heart diseases

\section{Background}

Cardiovascular disease (CVD) is the number one cause of death in the United States and other industrial nations. ${ }^{1}$ In 2004, there were about 2.4 million deaths in the United States, and of these, approximately $36 \%$ were attributed to CVDs such as stroke, acute myocardial infarction, atherosclerosis, CHD and pulmonary embolism. ${ }^{1}$ The economic impact of CVDs on the United States healthcare system continues to grow as the population ages. The estimated direct and indirect cost of CVD approached US $\$ 500$ billion in 2008. ${ }^{1}$ Thus, CVD is a very costly disease in terms of both human lives and economics. There have been major advances in strategies to decrease the incidence of CVD, one of which is controlling and improving the lipid profile of patients who are at risk.

The first significant study that associated lipid abnormalities with CVD risk was the Framingham Heart Study, which started in 1948. In this study, over ten thousand subjects

submit your manuscript | www.dovepress.con 
were enrolled and several risk factors for CVD were identified, including high blood pressure, high blood cholesterol, smoking, obesity, diabetes and physical inactivity. ${ }^{2}$ Since the results of the Framingham Heart Study were published, there have been many trials for each of the CVD risk factors, especially elevated serum cholesterol levels.

After evaluating the major studies of cholesterol and lipids, the National Cholesterol Education Program (NCEP) set up guidelines for cholesterol management, referred to as the Adult Treatment Panel (ATP). The most recent panel is ATP III, which was released in 2001 and updated in 2004. According to ATP III, the primary target of cholesterol treatment is LDL-C (commonly referred to as "bad cholesterol"). ${ }^{3}$ The LDL-C treatment goal depends on an individual patient's risk for CHD. ${ }^{3}$ The higher the risk for CHD, the lower the target LDL-C should be. For example, in high risk individuals (those who have already had CHD or who are at risk of CHD), LDL-C levels should be $<100 \mathrm{mg} / \mathrm{dL}$, while moderately high risk individuals ( 2 or more risk factors, 10 -year CHD risk of $10 \%$ to $20 \%$ ) should target their LDL-C to $<130 \mathrm{mg} / \mathrm{dL} .{ }^{3,4}$ If LDL-C does not reach the desired level, then therapeutic lifestyle changes (TLCs) such as physical activity and diet modification are recommended. Drug therapy can also be initiated. ${ }^{3}$

Only after LDL-C levels are achieved does ATP III recommend treating other lipids, such as non-high-density lipoproteins (non-HDL-C), which consist of LDL-C and very low-density lipoproteins (VLDL-C), as a secondary target in patients with TG levels of 200 to $499 \mathrm{mg} / \mathrm{dL} .{ }^{3}$ Drug therapy can only be used to increase HDL-C, also known as "good cholesterol," in patients who have metabolic syndrome and a history of CHD or the risk equivalent. ${ }^{3}$ The current recommendations for treating LDL-C as the primary target are supported by most of the guidelines for cholesterol management. ${ }^{4-8}$

However, recent data suggests that HDL-C may have a more important role in cholesterol management than is suggested by the guidelines. Furthermore, the issue of whether $\mathrm{TG}$ is an independent risk factor for CHD or should be one of the primary targets of therapy is controversial. To understand each side in the argument, the metabolism/transport of cholesterol and lipoproteins in dyslipidemia and its effect on CHD, as well as different therapeutic targets, including current and future drug therapies, will be discussed.

\section{Cholesterol and lipoprotein metabolism and transport}

The major lipids are cholesterol, TG, and phospholipids, ${ }^{9}$ which are used for cell membrane formation, hormone synthesis, ${ }^{10}$ and bile acid production. Since lipids are not soluble in blood, they are packaged into complexes of lipid and protein called lipoprotein particles. ${ }^{9}$ Lipoproteins are composed of phospholipids, free cholesterols, proteins (known as Apo lipoproteins), cholesterol esters, and TG. ${ }^{9}$ There are 5 major classes of lipoprotein particles: chylomicrons, VLDL-C, intermediate density lipoproteins, LDL-C, and HDL-C. ${ }^{9}$

Chylomicrons are the largest lipoprotein particles, and transport dietary TG and cholesterols ${ }^{11}$ from the intestine ${ }^{9}$ to different parts of the body. The metabolism of chylomicrons is mediated by lipoprotein lipase (LPL), with ApoC-II acting as a cofactor and activator. ${ }^{9,11}$ Chylomicrons are usually present in the plasma for 3-6 hours after eating and are metabolized after 10 to 12 hours of fasting. ${ }^{11}$ After LPL metabolizes the chylomicrons and removes a large portion of the TG, chylomicrons are further metabolized in the liver by hepatic lipase, returning ApoC-II to HDL-C.9,11 Chylomicron remnants, which contain Apo-E and ApoB-48, are then taken up by remnant receptors in the liver, which liberates free cholesterols intracellularly. ${ }^{9}$ During the whole process of chylomicron metabolism, some of the components of the chylomicron such as phospholipids and some Apo lipoproteins are recycled and used to make HDL-C. ${ }^{9}$

VLDL-C is synthesized in the liver in response to a high carbohydrate diet. Excess carbohydrate is converted into TG and transferred to nascent VLDL-C. VLDL-C delivers TG to adipose tissue and cardiac or skeletal muscle for storage and energy release by LPL. Almost all LDL-C is derived from VLDL-C. ${ }^{11}$

LDL-C carries about $60 \%$ to $70 \%$ of serum cholesterol. ${ }^{9}$ It transports cholesterol from the liver to peripheral tissues. High levels of LDL-C are harmful, as LDL-C can build up on arterial walls, leading to the formation of atherosclerotic plaques. The binding of LDL-C to its receptor in the liver is the major mechanism of removal of LDL-C from the circulation. ${ }^{12}$ An increase in intracellular cholesterol inhibits de novo synthesis of cholesterol, resulting in decreased synthesis of LDL-C receptor and increased activity of an enzyme that facilitates cholesterol storage. ${ }^{9}$

HDL-C is known to be protective against CVD, while low HDL-C levels increase the risk of CVD. HDL-C is produced in the intestine, liver and plasma as a complex of Apo A lipoproteins, phospholipids and cholesterol. In the plasma, HDL-C is converted to a cholesterol ester by the action of cholesterol ester transferase (LCAT). As they circulate in the blood stream, HDL-C particles acquire more cholesterol from the blood stream. In addition, HDL-C particles remove cholesterol through a reverse cholesterol transport process 
from peripheral tissues and atheroma within the arteries to the liver, carrying approximately $30 \%$ of the serum cholesterol. ${ }^{11}$ Women and individuals on estrogen therapy generally have higher HDL-C levels ${ }^{9}$ due to the effect of estrogen on the upregulation of the major components of the HDL-C particle, mainly ApoA-1 lipoprotein.

\section{HDL as a risk factor for heart disease}

The process known as reverse cholesterol transport involves removal by HDL-C of un-esterified (free) cholesterol from peripheral cells, such as macrophages, and delivery to the liver through the interaction of HDL-C with the hepatic HDL receptor. Several studies have shown an inverse relationship between blood HDL-C levels and heart disease. These studies show that individuals with low levels of HDL-C have worse CHD outcomes. Furthermore, data obtained from several epidemiological studies emphasize that the risk factor of low HDL-C levels is completely independent of LDL-C; ie, no matter how low the LDL-C level, a decrease in the HDL-C would increase the risk for coronary artery disease.

\section{Etiology of dyslipidemia and effects on atherosclerosis and CHD/CVD}

There are many causes of dyslipidemia, both primary and secondary. The primary causes of dyslipidemia are due mostly to genetic disorders. There are six categories of lipoprotein disorders according to the Fredrickson-Levy-Lees classification: Type I (high levels of chylomicrons), Type IIa (high LDL-C levels), Type IIb (high LDL-C and VLDL-C levels), Type III (high IDL-C levels), Type IV (high VLDL-C levels), and Type V (high LDL-C and chylomicrons levels). It is also possible that some disease states can be placed into more than one category of lipoprotein disorder.

The term hypertriglyceridemia usually refers to elevations in VLDL-C and chylomicrons, both of which carry and transport TG. ${ }^{5}$ Primary hypertriglyceridemia includes primary chylomicronemia, familial hypertriglyceridemia, familial combined hyperlipoproteinemia and familial dysbetalipoproteinsemia. ${ }^{12}$ Primary chylomicronemia is a genetic disease that is characterized by a deficiency in LPL or cofactor, and results in elevated chylomicrons and VLDL-C and severe elevation of TG, leading to acute pancreatitis. ${ }^{12}$ Familial hypertriglyceridemia is a Type IV disorder in which primarily VLDL-C is affected. ${ }^{9}$ It is caused by a number of genetic determinants that result in insufficient removal of TG-rich lipoproteins. ${ }^{12}$ Familial combined hyperlipoproteinemia is a disorder characterized by increased levels of VLDL-C, LDL-C, or both. Familial dysbeta lipoproteinemia is a disorder characterized by increased levels of VLDL-C remnant and chylomicron remnant. ${ }^{9,12}$

The term hypercholesterolemia usually refers to elevated serum LDL-C. Primary hypercholesterolemia includes familial hypercholesterolemia, familial ligand-defective Apo lipoprotein B, and familial combined hyperlipoproteinemia. Primary hypercholesterolemia is a Type IIa dominant disorder that involves mutations in the LDL-C receptor gene. Homozygotes usually have a worse prognosis than heterozygotes. Familial ligand-defective apoliporotein B is also a Type IIa disorder caused by a mutation in ApoB-100 that disrupts the binding of LDL-C to the LDL-C receptor, thereby decreasing metabolism of LDL-C. In both of these disorders, LDL-C receptor-mediated endocytosis in the liver is decreased, resulting in increased serum LDL-C. In addition, genetic evidence confirms the role of a newly discovered serine protease, pro-protein convertase subtilisin-like kexin type 9 (PCSK9), in patients suffering from autosomaldominant hypercholesterolemia. Three gain of function single nucleotide polymorphisms in the PCSK9 gene have been identified that increase LDL-C levels. PCSK9 destroys low density lipoproteins receptors (LDL-C-R) in the liver, thereby regulating the levels of LDL-C in plasma. Lastly, familial combined hyperlipoproteinemia is a Type IIb disorder that can cause elevated VLDL-C, LDL-C, or both.

Some rare genetic disorders can also cause low levels of HDL-C, ie, Tangier disease, disorders of LCAT and familial hypoalphalipoproteinemia. Tangier disease is caused by mutation and loss of function of ATP-binding cassette 1 (ABCA1). ABCA1 transports cholesterol and phospholipids out of cells for pickup by Apo-A1 in the circulation. In the absence of ABCA1, free Apo-A1 does not acquire cellular lipids. This results in accelerated clearance of Apo-A1 from plasma, leading to low HDL-C levels. ${ }^{13}$ LCAT is a lecithin cholesterol acyltransferase responsible for catalyzing the formation of cholesterol esters of HDL-C and LDL-C; thus, it is crucial for HDL-C formation. ${ }^{14}$ Familial hypoalphalipoproteinemia includes a wide range of disorders that result in low levels of HDL-C (usually below $35 \mathrm{mg} / \mathrm{dL}$ ).

There are many secondary causes of dyslipidemia, including disease- and medication-induced dyslipidemia. Some of the more common causes of hypertriglyceridemia are diabetes mellitus, alcohol abuse, estrogen, and obesity., ${ }^{9,12}$ Some of the more common causes of hypercholesterolemia are hypothyroidism, anorexia, and excess corticosteroid use. ${ }^{9,12}$ Low levels of HDL-C can be caused by malnutrition, obesity, and drugs such as beta-blockers, anabolic steroids, isotretinoin, and progestins. ${ }^{9}$ 
High levels of LDL-C (and to a lesser extent, VLDL-C) result in the accumulation of LDL-C in the arterial wall, leading to oxidation of LDL-C. ${ }^{9}$ Oxidized LDL-C can cause extensive damage to the arterial wall, provoking inflammation responses, promoting coagulation, increasing the activity of mediators that cause vasoconstriction and inhibiting mediators that cause vasodilation. ${ }^{9}$ Oxidized LDL-C recruits monocytes, which enter the arterial wall and are activated to become macrophages. The macrophages ingest oxidized LDL-C through the macrophage scavenger receptor to become foam cells, or fatty streak. Foam cells propagate inflammatory responses as well as facilitate deposition of more oxidized LDL-C. Micro-calcification of the vascular smooth muscle cells will take place, which progresses to atherosclerosis. The fatty streak, which consists of cholesterol-filled macrophages, is the first stage of atherosclerosis. Plaques (deposits of fatty substances, cholesterol, calcium, and cell components) will then form and progress, gradually increasing inside the artery and narrowing the arterial wall, resulting in decreased flow of blood and oxygen to tissues. Plaques are usually kept in check by a fibrous cap, which protects and stabilizes the lesion. If the plaque ruptures, then thrombosis will occur and damage will spread to other areas, leading to ischemic heart disease, myocardial infraction (MI), stroke, peripheral arterial disease, other CVDs, and possibly death. Therefore, it is crucial that dyslipidemia, primary or secondary, is treated.

Unlike LDL-C, HDL-C is believed to help protect against CVD. Its protective effects are believed to be due in part to reverse cholesterol transport. As the name suggests, the reverse cholesterol transport process involves the transport of HDL-C from peripheral tissues and transfer to VLDL-C and LDL-C back to liver for secretion in the bile. ${ }^{9} \mathrm{HDL}-\mathrm{C}$ is also believed to have anti-inflammatory, anti-oxidative, platelet antiaggregatory, anticoagulation, and profibrolytic effects, ${ }^{13}$ which can help reduce damage mediated by high levels of LDL-C.

Based on the current knowledge of LDL-C and HDL-C, it is generally accepted that low levels of LDL-C and high levels of HDL-C are ideal for any patient. However, the recommendation to target both of these lipoproteins as surrogate goals of therapy needs to be based on the results of clinical studies. The same can be said for the use of pharmacotherapy to lower TG levels.

\section{LDL-C and current guidelines for lipid management}

ATP III and its update are the current lipid management guidelines published by the NCEP. This guideline, along with most of the other lipid management guidelines, recommends that clinicians primarily target LDL-C in treating dyslipidemia. ${ }^{4-8}$ The LDL-C goal will depend on patient risk factors for CHD. According to ATP III, the risk factors for CHD are cigarette smoking, hypertension (>140/90 mmHg), low HDL-C $(<40 \mathrm{mg} / \mathrm{dL})$, family history of premature CHD (CHD in first degree relative, male $<55$ years old and female $<65$ years old), and age (male $>45$ years old and female $>55$ years old). ${ }^{3}$ People who have previously had CHD (including MI, angina, coronary artery procedures, and myocardial ischemia) or who have a CHD risk equivalent (history of diabetes, noncoronary atherosclerotic disease such as peripheral vascular disease, or 2 or more risk factors for CHD with a 10 year CHD risk of $>20 \%$ ) are considered high risk patients whose LDL-C goal should be $<100 \mathrm{mg} / \mathrm{dL}$ or $<70 \mathrm{mg} / \mathrm{dL}$. ${ }^{3,4}$

Moderately high risk refers to individuals with 2 or more risk factors and a 10 -year risk of $10 \%-20 \%{ }^{3}$ Moderate risk refers to individuals with 2 or more risk factors and a 10 -year risk $<10 \%$, while low risk refers to individuals with 0 to 1 risk factors. ${ }^{3}$ The LDL-C target for moderately high risk and moderate risk is $<130 \mathrm{mg} / \mathrm{dL}$; the LDL-C target for low risk is $<160 \mathrm{mg} / \mathrm{dL}^{3}$ TLCs, such as decreasing intake of cholesterol and saturated fat in the diet, increasing intake of plant stanols/sterols and viscous fiber, reducing weight and increasing physical activity are recommended whenever LDL-C levels are above target. ${ }^{3}$ Drug therapy is considered when LDL-C is above a certain level based on individual risk category. ${ }^{3}$

Non-HDL-C (LDL-C + VLDL-C) becomes the secondary target only if an individual's TG fall between 200 to $499 \mathrm{mg} / \mathrm{dL}^{3}$ It becomes the primary target after TG reach $500 \mathrm{mg} / \mathrm{dL}$, at which point the priority becomes the prevention of acute pancreatitis. ${ }^{3}$ Even though the NCEP currently considers an HDL-C of $<40 \mathrm{mg} / \mathrm{dL}$ as a risk factor (an increase from HDL-C $<35 \mathrm{mg} / \mathrm{dL}$ in ATP II), there is no specific goal recommended for HDL-C. Moreover, treatment of individuals with low HDL-C is only considered for high risk patients with metabolic syndrome, and only after LDL-C has been treated.

ATP III is based on evidence from a number of studies, most of which focus on HMG-CoA reductase inhibitors, also known as statins, which have the greatest effect on LDL-C. For example, in the Primary Prevention of Acute Coronary Events with Lovastatin in Men and Women with Average Cholesterol Levels trial, it was shown that lovastatin 20 to $40 \mathrm{mg}$ is better than placebo in decreasing acute coronary events in subjects with average total cholesterol (TC) and LDL-C, and low HDL-C. ${ }^{14}$ Since lovastatin had a greater effect on LDL-C (a 25\% decrease from baseline) 
than TC (18\% decrease), TG (15\% decrease), or HDL-C ( $6 \%$ increase), the $37 \%$ relative risk reduction in major acute coronary events (MI, unstable angina, sudden cardiac death) reported in this study ${ }^{14}$ can be attributed mostly to the decrease in LDL-C. The same can be said for several pravastatin trials, in which pravastatin $40 \mathrm{mg}$ daily was shown to be better than placebo for primary and secondary prevention of coronary events. ${ }^{15-17}$ Some trials have shown that statins not only decrease death from cardiovascular causes, but also all-cause mortality. ${ }^{15,17}$ However, since the most significant changes are observed in LDL-C levels, the bulk of the benefit of statins is attributed to the reduction in LDL-C.

The Scandinavian Simvastatin Survival Study (S4) examined the independent effects of LDL-C, HDL-C, TC, and TG on major coronary events. S4 showed that simvastatin 20-40 mg as compared to placebo reduces the relative risk of major coronary events by $34 \%$, coronary death by $42 \%$, and all cause death by $30 \%$ in subjects with angina or previous MI and relatively high TC. ${ }^{18}$ Further analysis showed that each additional $1 \%$ reduction in LDL-C reduces major coronary event (MCE) risk by $1.7 \%$ (CI $1.0 \%$ to $2.4 \%$ ); each $1 \%$ decrease in TC decreases MCE risk by $1.9 \%(1.0 \%$ to $2.8 \%$ ); each $1 \%$ increase in HDL-C decreases risk by $0.8 \%$ $(0.1 \%$ to $1.5 \%)$. No significant difference was associated with a $1 \%$ reduction in TG. ${ }^{19}$

In summary, ATP III contains evidenced-based recommendations supporting LDL-C as a primary target for therapy. Even though most of the clinical trials do not look at the independent effect of lowering LDL-C on CHD, most of the analysis supports an association between decreased LDL-C and reduced CHD incidence. ${ }^{18,20,21}$ Thus, the level of evidence that supports the recommendation of decreasing LDL-C to reduce CHD risk is moderately strong Grade A evidence based on randomized controlled trials. Despite the suggestion that the CHD morbidity/mortality and all-cause mortality benefits of LDL-C lowering drugs (especially statins) are due exclusively to LDL-C lowering, LDL-C should continue to be a primary target in treatment of patients with dyslipidemia.

\section{HDL-C}

There is an inverse relationship between HDL-C and CHD. Recent studies indicate that there is a high prevalence of individuals with low levels of HDL-C in the population. For example, in a European study of 8545 dyslipidemia patients, the prevalence of low levels of HDL-C $(<40 \mathrm{mg} / \mathrm{dL}$ in men and $<50 \mathrm{mg} / \mathrm{dL}$ in women) was $33 \%$ in men and $40 \%$ in women who were treated for dyslipidemia. ${ }^{22}$ In a nationwide
Mexican study including 15607 subjects aged 20 to 69 , it was shown that the prevalence of low HDL-C (below $0.9 \mathrm{mmol}$ or $35 \mathrm{mg} / \mathrm{dL}$ ) was approximately $46 \%$ for men and $29 \%$ for women. ${ }^{23}$ This high prevalence is problematic, because according to the Framingham 10 year CHD risk assessment (derived from the Framingham Heart Study), low HDL-C is linked to worse CHD outcomes. It is estimated that $>40 \%$ of coronary events occur in individuals with $<40 \mathrm{mg} / \mathrm{dL}$ HDL-C. Moreover, according to a 12-year cohort study following the Framingham population, multivariable-adjusted relative risk for CHD (using HDL-C of 35 to $59 \mathrm{mg} / \mathrm{dL}$ as a reference) is 1.47 for males and 2.02 for females with HDL-C $<35 \mathrm{mg} / \mathrm{dL}$, and 0.56 for males and 0.58 for females with HDL-C $>60 \mathrm{mg} / \mathrm{dL} .{ }^{24}$ Data from several epidemiological studies emphasize that the risk factor associated with low level HDL-C is totally independent of LDL-C; ie, no matter how low the LDL-C level, a decrease in the HDL-C level increases the risk of CHD.

Although many potential protective mechanisms for HDL-C have been proposed, the precise anti-atherogenic mechanism has yet to be resolved. The most widely accepted mechanism is reverse cholesterol transport. This process is mediated by HDL-C and involves the transfer of cholesterol from macrophages/foam cells present in peripheral tissues through the blood stream to the liver, with subsequent metabolism of cholesterol and secretion into the bile. ${ }^{9}$ Other potential mechanisms include maintenance of endothelial integrity through increased nitric oxide (a vasodilator) bioavailability, promotion of endothelial cell proliferation and migration, and prevention of endothelial cell apoptosis. ${ }^{25}$ Moreover, HDL-C has been shown to inhibit thrombosis, oxidation, ${ }^{25}$ and inflammation, ${ }^{26}$ all of which can contribute to atherosclerosis and CHD. However, despite current evidence of the potential benefits of HDL-C, clinical studies are needed to bolster the recommendation to target HDL-C in lipid management. This, however, can be a daunting task, because most drugs for lipid management affect more than one type of lipoprotein. That being said, several studies have analyzed the independent effects of different classes of lipoproteins on CHD. Table 1 summarizes the results of several randomized, controlled trials evaluating the effects of lipid drug therapy on CHD.

\section{Analysis of the Scandinavian Simvastatin Survival Study ${ }^{18}$}

The Scandinavian Simvastatin Survival Study was a randomized controled trial studying the effect of simvastatin 20 to $40 \mathrm{mg}$ on MCE such as acute MI, sudden cardiac death and 


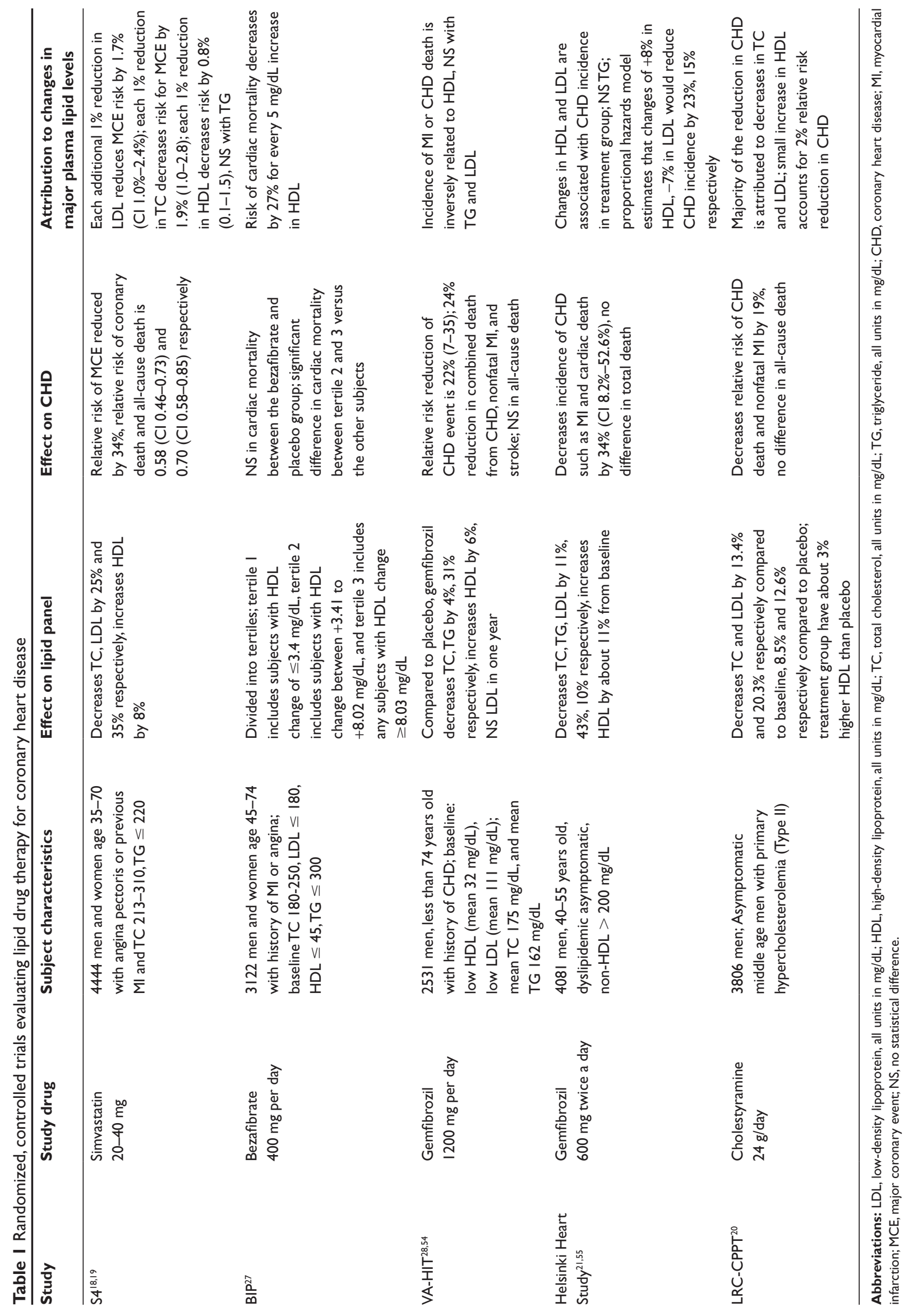


resuscitated cardiac arrest in 4,444 subjects with previous angina or MI. ${ }^{18}$ Simvastatin 20 to $40 \mathrm{mg}$ decreased TC and LDL-C by $25 \%$ and $35 \%$, respectively, and increased HDL-C by $8 \%$. The relative risk of MCE was reduced by $34 \%$, and the relative risk of coronary death and all-cause death was 0.58 (CI 0.46 to 0.73 ) and 0.70 (CI 0.58 to 0.85 ), respectively. ${ }^{18}$ An analysis of the relationship between lipoprotein changes and incidence of MCE showed that each additional $1 \%$ reduction in LDL-C reduced MCE risk by $1.7 \%$ (CI $1.0 \%$ to $2.4 \%$ ) and each $1 \%$ increase in HDL-C decreased risk by $0.8 \%$ (CI $0.1 \%$ to $1.5 \%$ ). ${ }^{19}$ Thus, the benefits of increasing HDL-C were small but statistically significant as compared to the benefits of decreasing LDL-C. However, one limitation of this study is that only baseline and first year lipoprotein and cholesterol measurements were taken into account; the study did not analyze long term changes in lipid profiles. Another point to consider is that statins in general exert other protective effects, including slightly increasing HDL-C, thus making it difficult to distinguish the effects of HDL-C on MCE.

\section{Analysis of the Bezafibrate Infarction Prevention (BIP) trial ${ }^{27}$}

The goal of the BIP trial was to determine the effect of bezafibrate on MCE (MI, sudden cardiac death) and mortality in 3122 subjects with a history of MI or angina. After the original study ended (median follow-up 6.2 years) and study medication was discontinued, the patients were followed for an additional 7.9 years. On-treatment lipid panels and cardiac mortality through the extended follow-up were used for analysis. The bezafibrate subjects were stratified into three tertiles based on changes in HDL-C from baseline: tertile 1 included subjects with HDL-C changes of less than or equal to $3.4 \mathrm{mg} / \mathrm{dL}$; tertile 2 included subjects with HDL-C changes between +3.41 to $+8.02 \mathrm{mg} / \mathrm{dL}$; and tertile 3 included any subjects with HDL-C changes greater than or equal to $8.03 \mathrm{mg} / \mathrm{dL}$. There was no statistical difference in cardiac mortality between the bezafibrate and placebo groups. However, when sub-divided into tertiles, there was a significant difference in cardiac mortality between tertile 2 and 3 versus the other groups. The study concluded that the risk of cardiac mortality is decreased by $27 \%$ for every $5 \mathrm{mg} / \mathrm{dL}$ increase in HDL-C.

\section{Analysis of the Veteran Affairs High-density Lipoproteins Intervention Trial (VA-HIT) $)^{28}$}

The VA-HIT trial was a randomized, double-blind, placebocontrolled trial designed to determine the effect of gemfibrozil on CHD events in men with a history of CHD who had low HDL-C (mean $32 \mathrm{mg} / \mathrm{dL}$ ) and low LDL-C (mean $111 \mathrm{mg} / \mathrm{dL}$ ). The VA-HIT trial showed that gemfibrozil $1200 \mathrm{mg}$ per day reduces major CHD events (MI and CHD death) by $22 \%$ as compared to placebo during a median follow-up of 5.1 years. The analysis looked at lipid levels at 4 through 18 months during the trial. The incidence of MI or CHD death was inversely related to HDL-C levels, and not related to TG and LDL-C. Some limitations of the study are that all the subjects were male and only lipid panels in the first 18 months were analyzed. In addition, changes in HDL-C levels in the first year ( $6 \%$ changes versus placebo) were rather small for a fibric acid derivative, leading to questions about compliance.

\section{Analysis of the Helsinki Heart study ${ }^{21}$}

The Helsinki Heart Study was a study of 4081 dyslipidemic men aged 40 to 55 who did not have CHD or other major disabilities such as mild hypertension or non-insulin-dependent diabetes. Gemfibrozil $600 \mathrm{mg}$ twice a day decreased the incidence of CHDs, such as MI and cardiac death by 34\% (CI 8.2\% to $52.6 \%$ ) without decreasing all-cause mortality. For the most part, lipid panel changes were responsible for the decrease in CHD. Changes in HDL-C and LDL-C were associated with CHD incidence in the treatment group while changes in TG were not. Estimates based on a proportional hazards model indicated that changes of $+8 \%$ in HDL-C and $-7 \%$ in LDL-C would reduce $\mathrm{CHD}$ incidence by $23 \%$ and $15 \%$, respectively. Thus, a $1 \%$ increase in HDL-C can bring about a $2 \%$ to $3 \%$ decrease in CHD. One limitation of this study is that the subjects were all male and employed by specific companies, which undermines extrapolation of the data.

\section{Analysis of the Lipid Research Clinics Coronary Primary Prevention Trial $\left(\right.$ LRC-CPPT) ${ }^{20}$}

The LRC-CPPT trial investigated the efficacy of cholestyramine $24 \mathrm{~g}$ per day in 3806 asymptomatic middle aged men with primary hypercholesterolemia (Type II). Cholestyramine decreased the relative risk of CHD death and nonfatal MI by $19 \%$ without significantly decreasing all-cause mortality. The investigators proposed that the decreases in TC and LDL-C accounted for the majority of the benefit, while the small increase in HDL-C (about 3\%) accounted for a $2 \%$ decrease in CHD.

\section{Systematic review of HDL-C and $\mathrm{CH}^{29}$}

A systematic review was performed by Dean et al on the effects of HDL-C on cardiovascular risk. ${ }^{29} \mathrm{~A}$ search for 
articles published between January 1965 and March 2001 yielded 51 articles for analysis (including 19 statin trials, 6 fibrate trials, 2 resin trials, and 1 resin-niacin trial). The authors concluded that there is no statistically significant association between increased HDL-C levels and CHD morbidity and mortality rates, although there is a trend in that direction for CHD mortality $(p=0.08)$. However, one limitation of this study is that most of the articles reviewed were trials of statins, which only moderately increase HDL-C (generally $<7 \%$ to $10 \%$ ). This makes it more difficult to distinguish the effects of HDL-C on CHD morbidity and mortality. Furthermore, a recent review raised the concern that not all HDL-Cs are equally efficacious in their ability to decrease atherosclerosis and $\mathrm{CHD},{ }^{30}$ which can explain the range of differences between changes in HDL-C values and changes in CHD events. Nevertheless, a 1\% increase in HDL-C is associated with anywhere from a 0.7 to $3 \%$ decrease in CHD events. In addition, increases in HDL-C are associated with better CHD outcomes, and the level of $\mathrm{HDL}-\mathrm{C}$ is an independent predictor of CHD. ${ }^{24}$ The evidence that increasing HDL-C decreases CHD is moderately strong Grade A/B evidence from randomized controlled trials and meta-analyses, and supports consideration of targeting HDL-C, particularly in subjects with low levels, as well as LDL-C reduction.

\section{TG}

TG, like cholesterol, are one of the major lipids in the body. They are made up of glycerol and free fatty acids, and found mostly in chylomicrons and VLDL-C. Several studies have shown a correlation between high levels of TG and CHD. ${ }^{31,32}$ However, it is unclear whether high levels of TG are an independent risk factor for CHD, and whether TG should be a target for therapy. The results of the Prospective Cardiovascular Munster (PROCAM) study support high levels of TG as an independent risk factor. The PROCAM study was a massive trial including 25,502 men and women who were assessed for risk factors for CHD, such as dyslipidemia, and followed for approximately 8 years to assess development of CHD. ${ }^{33}$ Multivariant analysis showed a significant ageadjusted correlation between major coronary events and TC, HDL-C, LDL-C, and log-transformed TG. ${ }^{33}$

A meta-analysis of 17 prospective studies also supports TG as an independent risk factor for $\mathrm{CHD}^{34}$ The univariant relative risk for CHD with a $1 \mathrm{mmol} / \mathrm{L}(89 \mathrm{mg} / \mathrm{dL})$ increase in TG was 1.32 (95\% CI 1.26-1.39) for men and 1.76 (95\% CI 1.50 to 2.07) for women. ${ }^{34}$ After adjusting for variables such as HDL-C, cholesterol, and other risk factors, the relative risk was $1.14(95 \%$ CI 1.05 to 1.28$)$ for men and 1.37 (95\% CI 1.13 to 1.66 ) for women. ${ }^{34}$ However, a reduction in $\mathrm{TG}$ of $89 \mathrm{mg} / \mathrm{dL}$ seems extreme considering that normal levels of TG should be $<150 \mathrm{mg} / \mathrm{dL}$, according to ATP III guidelines. Thus, lowering of TG to a more reasonable level may not yield significant differences.

The S4 and VA-HIT studies did not show a significant association between reduced TG and CHD. ${ }^{19,28}$ The Helsinki Heart Study, despite documenting a $43 \%$ reduction in $\mathrm{TG}$, concluded that an independent effect of TG was not relevant. ${ }^{35}$ Secondary analysis of the Multiple Risk Factor Intervention Trial, The Lipid Research Clinics Coronary Primary Prevention Trial, and The Lipid Research Clinics Prevalence and Mortality Follow-Up Study showed that TG levels in men do not predict CHD risk, although the same cannot be said of women. ${ }^{36}$ However, TG is a significant amplifier of CHD in the presence of high LDL-C and low HDL-C.

In summary, high levels of TG are associated with $\mathrm{CHD}$ risk, independent of other risk factors such as LDL-C and TC. However, the link is very weak, especially in men. The evidence for increasing TG as a means to decrease CHD is Grade A/B evidence based on randomized controlled trials and meta-analyses.

\section{Current drugs for lipid management}

There are several classes of drugs on the market for lipid management, including HMG-CoA reductase inhibitors (statins), bile acid sequestrants, niacin (nicotinic acid), fibric acid derivatives, and cholesterol absorption inhibitors. Each class of drug affects lipoproteins and TG to different degrees; therefore, specific classes of drugs are used as firstline therapy when trying to target different lipoproteins. For example, because statins have the greatest effect on LDL-C, they are usually used as first-line therapy for high levels of LDL-C. On the other hand, niacin and fibric acid are more effective in increasing HDL-C and decreasing TG than the other classes of drugs. Table 2 shows the effects of each of the classes of drugs on lipids/lipoproteins.

\section{HMG-CoA reductase inhibitors (statins)}

The statins are the most widely used class of drug in lipid management. HMG-CoA reductase is the rate-limiting enzyme of the mevalonate pathway of cholesterol synthesis. Inhibition of HMG-CoA reductase disrupts cholesterol biosynthesis, thereby lowering total cholesterol in the body. The primary reason why this class of drug is so widely used is that it not only improves lipid profiles and CHD morbidity 
Table 2 Lipid management drugs and their effects on lipid/lipoprotein

\begin{tabular}{|c|c|c|c|}
\hline Drug class & LDL & HDL & TG \\
\hline $\begin{array}{l}\text { HMG-CoA reductase } \\
\text { inhibitors (statins) }\end{array}$ & Decrease $18 \%-55 \%$ & Increase $5 \%-15 \%$ & Decrease $7 \%-30 \%$ \\
\hline Bile acid sequestrants ${ }^{3}$ & Decrease $15 \%-30 \%$ & Increase $3 \%-5 \%$ & No change or increase \\
\hline Niacin (nicotinic acid) ${ }^{3}$ & Decrease $5 \%-25 \%$ & Increase $15 \%-35 \%$ & Decrease $20 \%-50 \%$ \\
\hline Fibric acid derivatives ${ }^{3}$ & $\begin{array}{l}\text { Decrease } 5 \%-20 \% \text {, may increase } \\
\text { in patients with high TG }\end{array}$ & Increase $10 \%-20 \%$ & Decrease $20 \%-50 \%$ \\
\hline Ezetimibe $^{51}$ & Decrease about $18 \%$ & Increase about I\% & Decrease about $8 \%$ \\
\hline
\end{tabular}

Abbreviations: LDL, low-density lipoprotein; HDL, high-density lipoprotein; TG, triglyceride.

and mortality, but has also been shown to decrease all-cause mortality in several clinical trials. ${ }^{15,17,18}$

There are a number of statins on the market. They include atorvastatin $\left(\right.$ Lipitor $\left.^{\circledR}\right)$, simvastatin $\left(\right.$ Zocor $\left.^{\circledR}\right)$, pravastatin $\left(\right.$ Pravachol $\left.^{\circledR}\right)$, lovastatin $\left(\right.$ Mevacor $^{\circledR}$, Altocor $\left.{ }^{\circledR}\right)$, fluvastatin $\left(\right.$ Lescol $\left.^{\circledR}\right)$, and rosuvastatin $\left(\right.$ Crestor $\left.^{\circledR}\right)$. Some combination products including statins are atorvastatin/amlodipine (Caduet), simvastatin/ezetimibe (Vytorin ${ }^{\circledR}$ ), pravastatin/ aspirin (Pravigard ${ }^{\circledR} \mathrm{PAC}$ ), and niacin ER/lovastatin $\left(\right.$ Advicor $\left.{ }^{\circledR}\right)$. The oral bioavailability of statins is about $5 \%$ to $30 \%$ due to first pass effects, and the concentration of statins peaks about 1 to 4 hours after oral administration. ${ }^{11}$ The half-life of atorvastatin and rosuvastatin is about 20 hours, while that of the other statins is about 1 to 4 hours, which may account for the relatively higher potency of atorvastatin and rosuvastatin. ${ }^{11}$ All of the statins are metabolized by cytochrome P-450 enzymes, except for pravastatin. ${ }^{37}$ Table 3 shows typical doses needed to achieve a $30 \%$ to $40 \%$ reduction in LDL-C as well as administration guidelines and methods of metabolism for the different statins. The relationship between the dose of statin and its effects on lipid profile is not linear. In general, for each doubling of dose, there is an approximate $6 \%$ decrease in LDL-C. ${ }^{3}$

Statins are contraindicated in patients with unexplained or persistent increases in serum transaminase levels, hypersensitivity to statins or any of its components, active liver disease, and pregnancy/lactaction. ${ }^{37-42}$ Statins should be used with caution when combined with niacin and fibrate (a combination that would increase the risk of myopathy) and when used with drugs that can cause an interaction. ${ }^{37-42}$ Some of the adverse effects associated with statins are diarrhea, flatulence, heartburn, nausea, vomiting, headache, myalgia, myositis, rhabdomyolysis, and elevated liver enzymes. ${ }^{37-42}$ When taking a statin, the patient's lipid panel and liver function should be monitored periodically. ${ }^{37-42}$ Serum creatine kinase (CK) should be monitored in patients experiencing muscle pain or who are taking other drugs that can cause myopathy ${ }^{37-42}$ If patients experience muscle fatigue/weakness/ache and their $\mathrm{CK}$ is greater than 10 times the upper limit of normal, the statin should be discontinued as soon as possible. If CK is 3 to 10 times the upper limit of normal, then the dose should be decreased, if not discontinued. ${ }^{43}$ If patients only have muscle symptoms without increased CK, then symptoms and CK should be monitored closely. ${ }^{43}$

\section{Bile acid sequestrants or bile acid binding resins}

Bile acid sequestrants or bile acid binding resins are positively charged resins that bind to negatively-charged bile acids in the intestine and prevent their absorption. ${ }^{11}$ To compensate for the loss of bile acids, the liver increases the conversion of cholesterol to bile acids. The conversion of cholesterol to bile acids reduces cholesterol in the body, resulting in a drop in cholesterol levels in the blood. ${ }^{11}$ However, the resins might also increase the production of TG, which can be problematic in patients with TG levels $>250 \mathrm{mg} / \mathrm{dL} .{ }^{11}$

The current resins on the market are cholestyramine $\left(\right.$ Questran $\left.{ }^{\circledR}\right)$, colestipol $\left(\right.$ Colestid $\left.^{\circledR}\right)$, and colesevelam $\left(\right.$ Welchol $\left.^{\circledR}\right)$. These resins increase HDL-C by about 3\% to $5 \%$ and decrease LDL-C by about $15 \%$ to $30 \% .{ }^{3}$ However, when combined with statins, the resins can provide an additional $12 \%$ to $16 \%$ reduction in LDL-C. ${ }^{3}$ The usual daily dose of cholestyramine is 4 to $16 \mathrm{~g}$ (powder form) given orally in divided doses. ${ }^{44}$ The drug should be mixed with at least 2 to 3 ounces of water, other beverage, soup, or pulpy fruits before administration. ${ }^{44}$ Because cholestyramine can cause discoloration of teeth and erosion of enamel, it should not be held in mouth for a long period of time. ${ }^{44}$ The usual daily dose of colestipol is 5 to $20 \mathrm{~g}$ (powder or tablet form) given orally once daily or in divided doses. ${ }^{3}$ When administering colesipol with other drugs, other drugs should be taken at least 1 hour before or 4 hours after colesipol. ${ }^{45}$ The powder 
Table 3 Statin drug summary

\begin{tabular}{|c|c|c|c|}
\hline Drug & $\begin{array}{l}\text { Usual dose in } \mathrm{mg} \text { for } \\
30 \%-40 \% \text { reduction } \\
\text { in } \mathrm{LDL}^{56}\end{array}$ & Administration guidelines ${ }^{37-42}$ & Metabolism $^{37-42}$ \\
\hline Atorvastatin (Lipitor $^{\circledR}$ ) & 10 & po w or w/o food & P450 CYP 3A4 \\
\hline Simvastatin $\left(\right.$ Zocor $\left.^{\circledR}\right)$ & $20-40$ & po in evening & P450 CYP 3A4 \\
\hline Pravastatin $\left(\right.$ Pravachol $\left.^{\circledR}\right)$ & $40-80$ & $\begin{array}{l}\text { po } w \text { or w/o food at anytime; when given with } \\
\text { bile-acid-binding resin, give pravastatin I hour } \\
\text { before or } 4 \text { hour after resin }\end{array}$ & $\begin{array}{l}\text { Isomerization, hydroxylation, } \\
\text { oxidation, conjugation }\end{array}$ \\
\hline Lovastatin $\left(\right.$ Mevacor $\left.^{\circledR}\right)$ & $40-80$ & $\begin{array}{l}\text { po take with evening meal for maximum } \\
\text { bioavailability }\end{array}$ & $\begin{array}{l}\text { Hydrolysis, P450 } \\
\text { CYP 3A4 }\end{array}$ \\
\hline Fluvastatin $\left(\right.$ Lescol $\left.^{\circledR}\right)$ & 80 & $\begin{array}{l}\text { po take } w \text { or } w / o \text { food in the evening; if taken } \\
\text { with bile-acid resin, administer fluvastatin at } \\
\text { bedtime at least } 2 \text { hours after resin }\end{array}$ & $\begin{array}{l}75 \% \text { via } \mathrm{P} 450 \text { CYP } 2 \text { C } 9 \\
5 \% \text { via } 2 C 8,20 \% \text { via } 3 A 4\end{array}$ \\
\hline Rosuvastatin $\left(\right.$ Crestor $\left.^{\circledR}\right)$ & 5 & $\begin{array}{l}\text { po } w \text { or w/o food at any time; if taken with } \\
\text { aluminum and magnesium combination antacid, } \\
\text { take antacid } 2 \text { hour after rosuvastatin }\end{array}$ & P450 CYP 2 C9 \\
\hline
\end{tabular}

Abbreviations: LDL, low-density lipoprotein; po, oral; w, with; w/o, without.

form of colestipol should be mixed with at least 3 ounces of liquid, soup, hot cereal, or pulpy fruit before administration. ${ }^{45}$ The usual daily dose of colesevelam is 2.6 to $3.8 \mathrm{~g}$ (tablet form) given orally in single or divided doses. ${ }^{3}$ Colesevelam should be taken with a meal and some liquid. ${ }^{46}$

Some of the side effects of bile acid resins are constipation, flatulence, indigestion, and GI distress. ${ }^{3}$ Resins can also decrease absorption of drugs such as statins, niacin, levothyroxine, and digoxin. ${ }^{3}$ Therefore, other drugs should be taken about 1 hour before or 4 hours after the resins (except colesevelam, which has a minimal effect on these other types of drug). ${ }^{3}$ Bile acid resins are contraindicated when TG is $>400 \mathrm{mg} / \mathrm{dL}$ or if the patient has familial dysbeta-lipoproteinemia, and should be used with caution when TG $>200 \mathrm{mg} / \mathrm{dL} .^{3}$

\section{Nicotinic acid or niacin}

Niacin is a water-soluble B vitamin that has anti-hyperlipidemic effects when given in large amounts. In general, niacin decreases LDL-C by about $5 \%$ to $25 \%$, increases HDL-C by $15 \%$ to $35 \%$, and decreases TG by $20 \%$ to $50 \%$. ${ }^{3}$ The mechanism of action of niacin is still unclear, but is thought to be mediated by a Gi coupled receptor (GPR109A or HM74A). It is postulated that niacin inhibits lipolysis of TG, which would reduce TG synthesis due to higher levels of TG in the body. ${ }^{11}$ Reduced TG in turn decreases VLDL-C and therefore LDL-C formation. Niacin also increases HDL-C by reducing ApoA-I clearance. ${ }^{11}$

In a class by itself, niacin comes in several formulations, including immediate release (IR), sustained release (SR; Slo-Niacin ${ }^{\circledR}$ ), and extended release $\left(\right.$ ER; Niaspan ${ }^{\circledR}$ ) products.
The usual dose of niacin is 1.2 to $3 \mathrm{~g}$ per day for IR, 1 to $2 \mathrm{~g}$ per day for SR, and 1 to $2 \mathrm{~g}$ per day for ER in single or divided doses. ${ }^{3}$ IR, SR, and ER products should not be substituted for each other because they are not the same; indeed, SR niacin has higher risk of liver toxicity than the other two formulations. ${ }^{3}$ Niacin should be administered orally at bedtime with a low-fat snack to decrease gastrointestinal irritation. ${ }^{47}$ The side effects of niacin include flushing, pruritus, nausea, vomiting, gastrointestinal irritation, and rare hepatotoxicity. ${ }^{47}$ Niacin can also cause an approximate 5\% increase in blood glucose levels and a $10 \%$ increase in uric acid levels. ${ }^{3}$ Aspirin or NSAID can be given 30 minutes before niacin to decrease flushing, pruritus, and gastrointestinal irritation.$^{47}$ Niacin is contraindicated in patients with active liver disease, active peptic ulcer, arterial bleeding, and hypersensitivity to niacin products. ${ }^{47}$ It should be used with caution in patients who are on concomitant anticoagulants or dilators, have diabetes, consume a large amount of alcohol, have a history of liver disease, renal disease, or unstable angina, or are predisposed to gout. ${ }^{47}$ While on niacin, lipid profile, blood glucose levels, liver function, serum phosphorus, and prothrombin time/INR (if on anticoagulation therapy) should be monitored. ${ }^{47}$

\section{Fibric acid derivatives}

The mechanism of action of fibric acid derivatives is still unclear, although some researchers believe that they activate peroxisome proliferator activated receptors (PPARs), which regulate gene transcription and influence blood lipid levels. ${ }^{48}$ The fibric acid derivatives that are currently on the market are gemfibrozil $\left(\right.$ Lopid $\left.^{\circledR}\right)$ and fenofibrate $\left(\right.$ Ticor $\left.^{\circledR}\right)$. The usual 
dose of gemfibozil is $600 \mathrm{mg}$ twice daily ${ }^{3}$ and the usual dose of fenobibrate is 48 to $145 \mathrm{mg}$ daily. ${ }^{49}$ These drugs decrease LDL-C by approximately $5 \%$ to $20 \%$, increase HDL-C by $10 \%$ to $20 \%$, and decrease TG $20 \%$ to $50 \%{ }^{3}$ Fibric acids may increase LDL-C in patients with high TG, thus, they should not be used in patients with high LDL-C, but may be useful for people suffering from metabolic syndrome with high TG, low HDL-C, and low LDL-C. Fenofibrate should be taken 1 hour before or 4 to 6 hours after bile acid resin, ${ }^{49}$ and gemfibrozil should be taken 30 minutes before morning and evening meals. ${ }^{50}$ Some of the side effects of fibrate therapy are dyspepsia, myopathy, gallstones, and increased liver function test; therefore liver function test should be monitored at baseline, 12 weeks into therapy, and periodically afterwards. ${ }^{3}$

\section{Cholesterol absorption inhibitors}

Cholesterol absorption inhibitors are a new class of drug whose mechanism of action involves inhibiting dietary cholesterol absorption. Ezetimibe $\left(\right.$ Zetia $\left.^{\circledR}\right)$ is currently the only drug available in this class. Ezetimibe alone can decrease LDL-C by about $18 \%$, increase HDL-C by $1 \%$, and decrease TG by $8 \% .{ }^{51}$ However, when combined with a statin (Vytorin), ezetimibe induced greater than expected changes in lipid profiles. ${ }^{51}$ A recent study involving 720 patients with very high levels of cholesterol due to an inherited form of heart disease examined whether Vytorin could slow the growth of plaques in carotid arteries more than simvastatin alone. Even though Vytorin dramatically reduced LDL-C levels, it did not slow the progression of artery blockage more than generic zocor.

The usual dose of ezetimibe is $10 \mathrm{mg}$ orally once daily with or without food. ${ }^{52}$ When administered with a bile acid resin, it should be taken at least 2 hours before or 4 hours after the resin. ${ }^{52}$ Some side effects of ezetimibe are diarrhea, abdominal pain, arthralgia, backache, myalgia, headache, sinusitis, hepatitis, anaphylaxis, myopathy and rhabdomyolysis (rare). ${ }^{52}$ Ezetimibe is contraindicated in active liver disease or persistently elevated liver enzyme (when taken with a statin). ${ }^{52}$ Caution should be used when giving ezetimibe to children under 10 years old and patients with hepatic insufficiency. ${ }^{52}$ While on ezetimibe, lipid panels should be monitored. Monitoring of serum CK is recommended in patients complaining of muscle pain or who are at high risk of developing myopathy or rhabdomyolysis. ${ }^{52}$

\section{Future drug therapy}

Many studies are underway to identify better drugs for treating abnormal lipid levels. Since the statins have proven to be the drug of choice for decreasing LDL-C, the focus of future lipid management therapy is to find new agents that increase HDL-C with minimal side effects. One potential mechanism of increasing HDL-C is inhibition of cholesteryl ester transfer protein (CETP). CETP is synthesized in the liver, and transfers cholesteryl esters from HDL-C to LDL-C or TG-rich lipoproteins in exchange for TG. ${ }^{11}$ After addition of TG to HDL-C, HDL-C is more readily catabolized in the liver. ${ }^{11}$ Partial inhibition of CETP is associated with an increase in HDL-C of up to $100 \%$, and may also decrease LDL-C levels. Current CETP inhibitors in development are JTT-705, torcetrapib, ${ }^{11}$ and anacetrapib. Clinical trials of torcetrapib have been terminated due to increased mortality associated with the torcetrapib/atorvastatin group as compared to the atrovastatin group in Phase 3 studies. The reason for this increase in mortality is unclear; however, certain side effects such as increased blood pressure have been noted. Since the three drugs employ different mechanisms to inhibit CETP, JTT-705 and anacetrapib still have the potential to be marketed.

Another potential target for raising HDL-C levels is augmenting ApoA-I levels (an important protein in HDL-C). Overexpression of ApoAI or infusion of ApoA-I in animal models increases HDL-C levels and decreases atherosclerosis. The two main ApoA-I peptides currently under investigation are the ApoA-I $\mathrm{I}_{\text {Milano }}$ complex and the D-4F peptide. Other possible targets include PPAR agonists, such as the glitazones, which are known to have modest HDL-C-boosting effects, although their main action is in reducing insulin resistance. PPAR agonists have been postulated to increase macrophage cholesterol efflux through increased levels of ABCA1 and ABCG1. Currently, niacin is considered the most effective pharmacologic agent for raising HDL-C. While the molecular mechanism of niacin's effect is not known, a G protein-coupled receptor called GPR109A (HM74A) has been identified as a niacin receptor. It is expressed primarily in adipocytes and its activation results in reduced release of fatty acids from adipose. This may explain niacin's effects on plasma TG, but fails to explain its effects on HDL-C. Compounds that specifically target the activation of GPR109A are currently in clinical trials. Finally, inhibition of endolipase, which hydrolyzes HDL-C and decreases HDL-C levels, is another potential approach. ${ }^{53}$

\section{Conclusion}

Cardiovascular disease is the number one cause of death in the United States. ${ }^{1}$ Since the Framingham Heart Study, which identified high cholesterol as one of the risk factors for CHD, there have been many trials studying the effect of changes 
in lipid profiles on CHD. These studies have also elucidated the functions, metabolism, and transport of lipids as part of normal physiology. Many primary and secondary causes of dyslipidemia have been identified. We now know that high LDL-C levels are associated with increased risk of $\mathrm{CHD}$, and high HDL-C levels are associated with reduced risk of CHD. The current ATP III guidelines for lipid management target LDL-C as the primary surrogate goal for lipid management. This recommendation is supported by many clinical trials. However, the ATP III guideline neglects to identify HDL-C as a target for therapy. Analysis of major randomized control trials shows that each $1 \%$ increase in HDL-C is associated with a $0.7 \%$ to $3 \%$ decrease in CHD. ${ }^{19-21,27,28}$ Thus, the risk factor associated with low HDL-C should be considered as an major independent risk factor for $\mathrm{CHD}^{29}$

Unlike LDL-C and HDL-C, the effects of reduced TG levels alone on CHD are less apparent, but reduced TG may be an important amplifier in the presence of dyslipedmic factors. Even though elevated TG might be an independent risk factor for CHD, a large reduction in TG is needed to show a difference, especially in the male population. New treatments using a combination of drugs targeting both LDL-C and HDL-C along with TG management would have a superior outcome for dyslipidemia therapy and decrease mortality due to $\mathrm{CHD} / \mathrm{CVD}$.

\section{Disclosures}

The authors disclose no conflicts of interest.

\section{References}

1. Disease Statistics. 2007 NHLBI Fact Book, Chapter 4. Available at: http://www.nhlbi.nih.gov/about/factbook/chapter4.htm. Accessed 27 May, 2008.

2. Framingham Heart Study: Design, Rationale, and Objectives. September 20; Available at: http://www.nhlbi.nih.gov/about/ framingham/design.htm. Accessed May 27, 2008.

3. Third report of the National Cholesterol Education Program (NCEP) Expert Panel on Detection, Evaluation, and Treatment of High Blood Cholesterol in Adults (Adult Treatment Panel III). Bethesda: US Department of Health and Human Services, Public Health Service, National Institutes of Health, National Heart, Lung and Blood Institute; 2001.

4. Grundy SM, Cleeman JI, Merz CN, et al. Implications of recent clinical trials for the National Cholesterol Education Program Adult Treatment Panel III guidelines. Circulation. 2004;110(2):227-239.

5. Fletcher B, Berra K, Ades P, et al. Managing abnormal blood lipids: a collaborative approach. Circulation. 2005;112(20):3184-3209.

6. VA/DoD clinical practice guideline for the management of dyslipidemia. Available at: http://www.guideline.gov.

7. Lipid management in adults. Available at: http://www.guideline.gov.

8. McCrindle BW, Urbina EM, Dennison BA, et al. Drug therapy of high-risk lipid abnormalities in children and adolescents: a scientific statement from the American Heart Association Atherosclerosis, Hypertension, and Obesity in Youth Committee, Council of Cardiovascular Disease in the Young, with the Council on Cardiovascular Nursing. Circulation. 2007;115(14):1948-1967.
9. Talbert RL. Hyperlipidemia. In: DiPiro JT, Talbert RL, Yee GC, Matzko GR, G. Wells B, Posey LM, editors. Pharmacotherapy: A Pathophysiologic Approach. 6th ed. New York: McGraw-Hill; Medical Publishing Division; 2005.

10. Dietschy JM. Dietary fatty acids and the regulation of plasma low density lipoprotein cholesterol concentrations. J Nutr. 1998; 128(2 Suppl):444S-448S.

11. Mahley RW, Bersot TP. Chapter 35: Drug therapy for hypercholesterolemia and dyslipidemia. In: Brunton LL, editors. Goodman and Gilman's The Pharmacological Basis of Therapeutics. 11th edition. New York: McGraw-Hill Companies, Inc.; 2006.

12. Malloy MJ, Kane JP. Agents used in hyperlipidemia. In: Katzung BG. Basic and Clinical Pharmacology. 9th ed. New York: The McGraw-Hill Companies, Inc.; 2004:561-575.

13. Nofer JR, Kehrel B, Fobker M, Levkau B, Assmann G, von Eckardstein A. HDL and arteriosclerosis: beyond reverse cholesterol transport. Atherosclerosis. 2002;161(1):1-16.

14. Downs JR, Clearfield M, Weis S, et al. Primary prevention of acute coronary events with lovastatin in men and women with average cholesterol levels: results of AFCAPS/TexCAPS. Air Force/Texas Coronary Atherosclerosis Prevention Study. JAMA. 1998;279(20):1615-1622.

15. Prevention of cardiovascular events and death with pravastatin in patients with coronary heart disease and a broad range of initial cholesterol levels. The Long-Term Intervention with Pravastatin in Ischaemic Disease (LIPID) Study Group. N Engl J Med. 1998;339(19):1349-1357.

16. Sacks FM, Pfeffer MA, Moye LA, et al. The effect of pravastatin on coronary events after myocardial infarction in patients with average cholesterol levels. Cholesterol and Recurrent Events Trial investigators. N Engl J Med. 1996;335(14):1001-1009.

17. Shepherd J, Cobbe SM, Ford I, et al. Prevention of coronary heart disease with pravastatin in men with hypercholesterolemia. West of Scotland Coronary Prevention Study Group. N Engl J Med. 1995;333(20):1301-1307.

18. Randomised trial of cholesterol lowering in 4444 patients with coronary heart disease: the Scandinavian Simvastatin Survival Study (4S). Lancet. 1994;344(8934):1383-1389.

19. Pedersen TR, Olsson AG, Faergeman O, et al. Lipoprotein changes and reduction in the incidence of major coronary heart disease events in the Scandinavian Simvastatin Survival Study (4S). Circulation. 1998;97(15):1453-1460.

20. The Lipid Research Clinics Coronary Primary Prevention Trial results. II. The relationship of reduction in incidence of coronary heart disease to cholesterol lowering. JAMA. 1984;251(3):365-374.

21. Manninen V, Elo MO, Frick MH, et al. Lipid alterations and decline in the incidence of coronary heart disease in the Helsinki Heart Study. JAMA. 1988;260(5):641-651.

22. Bruckert E, Baccara-Dinet M, McCoy F, Chapman J. High prevalence of low HDL-cholesterol in a pan-European survey of 8545 dyslipidaemic patients. Curr Med Res Op. 2005;21(12):1927-1934.

23. Aguilar-Salinas CA, Olaiz G, Valles V, et al. High prevalence of low HDL cholesterol concentrations and mixed hyperlipidemia in a Mexican nationwide survey. J Lipid Res. 2001;42(8):1298-1307.

24. Wilson PW, D'Agostino RB, Levy D, Belanger AM, Silbershatz H, Kannel WB. Prediction of coronary heart disease using risk factor categories. Circulation. 1998;97(18):1837-1847.

25. Mineo C, Deguchi H, Griffin JH, Shaul PW. Endothelial and antithrombotic actions of HDL. Circ Res. 2006;98(11):1352-1364.

26. Barter PJ, Nicholls S, Rye KA, Anantharamaiah GM, Navab M, Fogelman AM. Antiinflammatory properties of HDL. Circ Res. 2004;95(8):764-772.

27. Goldenberg I, Goldbourt U, Boyko V, Behar S, Reicher-Reiss H. Relation between on-treatment increments in serum high-density lipoprotein cholesterol levels and cardiac mortality in patients with coronary heart disease (from the Bezafibrate Infarction Prevention trial). Am J Cardiol. 2006;97(4):466-471.

28. Robins SJ, Collins D, Wittes JT, et al. Relation of gemfibrozil treatment and lipid levels with major coronary events: VA-HIT: a randomized controlled trial. JAMA. 2001;285(12):1585-1591. 
29. Dean BB, Borenstein JE, Henning JM, Knight K, Merz CN. Can change in high-density lipoprotein cholesterol levels reduce cardiovascular risk? Am Heart J. 2004;147(6):966-976.

30. Sviridov D, Mukhamedova N, Remaley AT, Chin-Dusting J, Nestel P. Antiatherogenic functionality of high density lipoprotein: how much versus how good. J Atheroscler Thromb. 2008;15(2):52-62.

31. Assmann G, Schulte H, Funke H, von Eckardstein A. The emergence of triglycerides as a significant independent risk factor in coronary artery disease. Eur Heart J. 1998;19 Suppl M:M8-M14.

32. Austin MA, Hokanson JE, Edwards KL. Hypertriglyceridemia as a cardiovascular risk factor. Am J Cardiol. 1998;81(4A):7B-12B.

33. Assmann G, Cullen P, Schulte H. The Munster Heart Study (PROCAM). Results of follow-up at 8 years. Eur Heart J. 1998;19 Suppl A: A2-A11.

34. Hokanson JE, Austin MA. Plasma triglyceride level is a risk factor for cardiovascular disease independent of high-density lipoprotein cholesterol level: a meta-analysis of population-based prospective studies. J Cardiovasc Risk. 1996;3(2):213-219.

35. Tenkanen L, Pietila K, Manninen V, Manttari M. The triglyceride issue revisited. Findings from the Helsinki Heart Study. Arch Int Med. 1994;154(23):2714-2720.

36. Avins AL, Neuhaus JM. Do triglycerides provide meaningful information about heart disease risk? Arch Int Med. 2000;160(13): 1937-1944.

37. Pravastatin sodium. Available at: http://www.thomsonhc.com. Accessed June 16, 2008.

38. Atorvastatin calcium. Available at: http://www.thomsonhc.com. Accessed June 16, 2008.

39. Simvastatin. Available at: http://www.thomsonhc.com. Accessed June 16, 2008.

40. Lovastatin. Available at: http://www.thomsonhc.com. Accessed June 16, 2008.

41. Fluvastatin sodium. Available at: http://www.thomsonhc.com. Accessed June 16, 2008.

42. Rosuvastatin calcium. Available at: http://www.thomsonhc.com Accessed June 16, 2008.
43. Pasternak RC, Smith SC Jr. Bairey-Merz CN, Grundy SM, Cleeman JI, Lenfant C. ACC/AHA/NHLBI clinical advisory on the use and safety of statins. J Am Coll Cardiol. 2002;40(3):567-572.

44. Cholestyramine. Available at: http://www.thomsonhc.com. Accessed June 16, 2008.

45. Colestipol hydrochloride. Available at: http://www.thomsonhc.com. Accessed June 16, 2008.

46. Colesevelam hydrochloride. Available at: http://www.thomsonhc.com. Accessed June 16, 2008.

47. Niacin. Available at: http://www.thomsonhc.com. Accessed June 16, 2008.

48. Kersten S, Desvergne B, Wahli W. Roles of PPARs in health and disease. Nature. 2000;405(6785):421-424.

49. Fenofibrate. Available at: http://www.thomsonhc.com. Accessed June 16, 2008.

50. Gemfibrozil. Available at: http://www.thomsonhc.com. Accessed June 16, 2008.

51. Zetia (ezetimibe) tablet. Feb 2008; Available at: http://dailymed.nlm. nih.gov/dailymed/drugInfo.cfm?id=7380. Accessed June 16, 2008.

52. Ezetimibe. Available at: http://www.thomsonhc.com. Accessed June 16, 2008.

53. Hausenloy DJ, Yellon DM. Targeting residual cardiovascular risk: raising high-density lipoprotein cholesterol levels. Heart. 2008;94(6): 706-714.

54. Rubins HB, Robins SJ, Collins D, et al. Gemfibrozil for the secondary prevention of coronary heart disease in men with low levels of high-density lipoprotein cholesterol. Veterans Affairs High-Density Lipoprotein Cholesterol Intervention Trial Study Group. NEngl J Med. 1999;341(6):410-418.

55. Frick MH, Elo O, Haapa K, et al. Helsinki Heart Study: primaryprevention trial with gemfibrozil in middle-aged men with dyslipidemia. Safety of treatment, changes in risk factors, and incidence of coronary heart disease. N Engl J Med. 1987;317(20):1237-1245.

56. Richmond $\mathrm{C}$. Cholesterol management. The Rx Consultant. 2006;15(2).
Vascular Health and Risk Management

\section{Publish your work in this journal}

Vascular Health and Risk Management is an international, peerreviewed journal of therapeutics and risk management, focusing on concise rapid reporting of clinical studies on the processes involved in the maintenance of vascular health; the monitoring, prevention and treatment of vascular disease and its sequelae; and the involvement of

\section{Dovepress}

metabolic disorders, particularly diabetes. This journal is indexed on PubMed Central and MedLine. The manuscript management system is completely online and includes a very quick and fair peer-review system, which is all easy to use. Visit http://www.dovepress.com/ testimonials.php to read real quotes from published authors. 